\section{Meta-analysis of the prevalence of physical inactivity among Brazilian adolescents}

\author{
Meta-análise de prevalência de inatividade física \\ entre adolescentes brasileiros
}

\author{
${ }^{1}$ Instituto de Estudos em \\ Saúde Coletiva, Universidade \\ Federal do Rio de Janeiro, \\ Rio de Janeiro, Brasil. \\ 2 Escola Nacional de Saúde \\ Pública Sergio Arouca, \\ Fundação Oswaldo Cruz \\ Rio de Janeiro, Brasil. \\ Correspondence \\ L. A. Barufaldi \\ Instituto de Estudos em Saúde \\ Coletiva, Universidade Federal \\ do Rio de Janeiro. \\ Av. Horácio Macedo s/n, Cidade \\ Universitária, Rio de Janeiro, \\ RJ 21941-598, Brasil. \\ laurabarufaldi@yahoo.com.br
}

\section{Abstract}

A systematic review and meta-analysis were carried out to investigate the prevalence of physical inactivity among adolescents in Brazil. We identified articles that had been published up to August 2010 and the search was conducted using six electronic databases. We did not enforce any search limitations. Forest plot-type graphs were generated using the prevalence of physical inactivity stratified by region and sex. Meta-regression models were fitted to identify possible sources of heterogeneity in the prevalence estimates. Of the 1,496 articles initially identified, 37 were deemed appropriate for the systematic review. Prevalence rates ranged from $2 \%$ to $80 \%$ for male and from $14 \%$ to $91 \%$ for female subgroups. The lowest prevalence rates of physical inactivity were found to be for the Southern region, whereas the highest rates were observed in the North-Northeast. The methods employed to evaluate physical inactivity in Brazilian adolescents also differed among the studies. This variation demonstrates the need for standardised and validated methods of measuring physical activity in epidemiological investigation.

Motor Activity; Adolescent; Meta-Analysis
Laura Augusta Barufaldi 1 Gabriela de Azevedo Abreu 1 Evandro Silva Freire Coutinho 2 Katia Vergetti Bloch 1

\section{Introduction}

Childhood and adolescence are extremely important periods for the development of a healthy lifestyle because the behaviors acquired throughout these stages tend to be perpetuated along the course of a lifetime ${ }^{1}$. Health-risk behaviors, such as physical inactivity, low fruit consumption, alcoholic-beverage consumption and tobacco use are increasingly present in our society and are associated with the development of chronic non-communicable diseases (NCDs) 2,3. Moreover, NCDs are increasingly prevalent among children and adolescents and are the leading cause of morbidity and mortality in Brazil as well as worldwide. NCDs can have serious personal, social and financial impacts 4,5 . Physical activity and healthy eating are two highpriority behaviors for the promotion of health and the prevention of NCDs 4 .

The most comprehensive study on physical activity was conducted between 2002 and 2003, with over 200,000 adults (18-69 years of age) from 51 countries (mostly of low to middle income), including Brazil ${ }^{6}$. Although the benefits of physical activity in relation to health are well established in the literature 7 , this study observed an average prevalence of physical inactivity of $18 \%$ (unweighed, i.e., for all countries), whereas the prevalence rates in Brazil were 25\% and 30\% for men and women, respectively 6 . 
In Brazil, several studies have examined physical activity. However, most of these studies have involved specific populations, i.e., representative samples of municipalities or states, not nationwide surveys.

According to a systematic review published in 2009, which described articles on the prevalence of physical activity (or inactivity) in Brazil, the number of studies on this topic has largely increased since 2000. Also, according to this review, most studies were conducted with adults. Among the studies that evaluated adolescents, the prevalences of physical inactivity were discrepant, ranging between $30 \%$ and $70 \% 8$.

Considering the importance of physical activity among adolescents and the dispersed information from Brazilian surveys, this article aims to describe the prevalence of physical inactivity among Brazilian adolescents through a systematic review and a meta-analysis of studies on this topic.

\section{Methodology}

This systematic review and meta-analysis has sought to identify articles published up to August of 2010 that were searchable on the following electronic databases: MEDLINE (http://www. ncbi.nlm.nih.gov/pubmed/), SciELO (http:// www.scielo.org), LILACS (http://lilacs.bvsalud. org/), Scopus (http://www.scopus.com/home. url), Web of Science (http://apps.webofknowl edge.com) and Adolec (http://www.adolec.br/ php/index.php). References of said articles were also searched to identify studies that were not found in the original search.

The descriptors used in the review process were selected after consulting the DeCs (Health Sciences Descriptors, BIREME) and MeSH (Medical Subject Headings, PubMed) terms. The search was conducted in Portuguese and/or English (depending upon the database) using blocks of three concepts: the first with terms related to physical activity ("physical activity", "exercise", "fitness", "motor activity", "physical inactivity" and "sedentarism"), the second with terms related to adolescence ("adolescent" and "young") and the third with terms related to Brazil ("Brazil”, "Brazilian" and each state separately). We used the logical operator "OR" to combine the descriptors within each block and the logical operator "AND" to combine the blocks. We also used a resource for term truncations, when necessary. We did not use search limits for the data, language, studydesign or sample size.

The inclusion criteria were as follows: (a) the sample population included adolescents (10 to
20 years of age) and the data for adolescents were presented separately if other age groups were included, (b) an assessment of physical inactivity was conducted, (c) data collection was performed in Brazil. We excluded reviews or studies that exclusively included adolescents with specific health conditions (obesity, hypertension, diabetes, etc.). We chose not to include theses, dissertations and monographs because a systematic search for these works was not logistically feasible.

Articles were first selected by their title and abstract and then by reading the pre-selected articles in full. Each paper was reviewed and selected by two reviewers (L.A.B. and G.A.A.) and, when there was a disagreement, a third person was consulted (K.V.B.). In cases in which there was more than one publication using the data from the same study, the most comprehensive article was selected.

In addition to the prevalence of physical inactivity, we recorded information on the location and time period of the data collection, type of population, assessment method and classification of physical activity.

The heterogeneity between the studies was assessed using the $\mathrm{I}^{2}$ statistic ${ }^{9}$. In order to obtain the summary measures, we used random-effect models due to the substantial heterogeneity of the results. In order to combine the prevalence measures, logit transformations were made initially to address the asymmetrical distribution. These prevalences were weighted by the inverse of the variance of the logit. Forest plot-type graphs were constructed using the prevalence of physical inactivity by region and by sex. Metaregression models were adjusted to identify possible sources of heterogeneity among the prevalences. The variables considered for this analysis were region, age, percentage by gender, type of population, sampling, sample size, method of evaluation and cut-off point.

The analyses were performed using Stata version 10.0 (Stata Corp., College Station, USA) and StatsDirect 2.7.8b (StatsDirect Ltd., Altrincham, UK).

\section{Results}

Figure 1 demonstrates the flowchart for the article selection process. Of the 1,496 articles initially identified (after removing duplicates), 37 were deemed eligible for the systematic review. The main objective of 18 of these articles was to analyse the prevalence of physical inactivity among adolescents. The remaining 19 articles had different primary goals although physical 


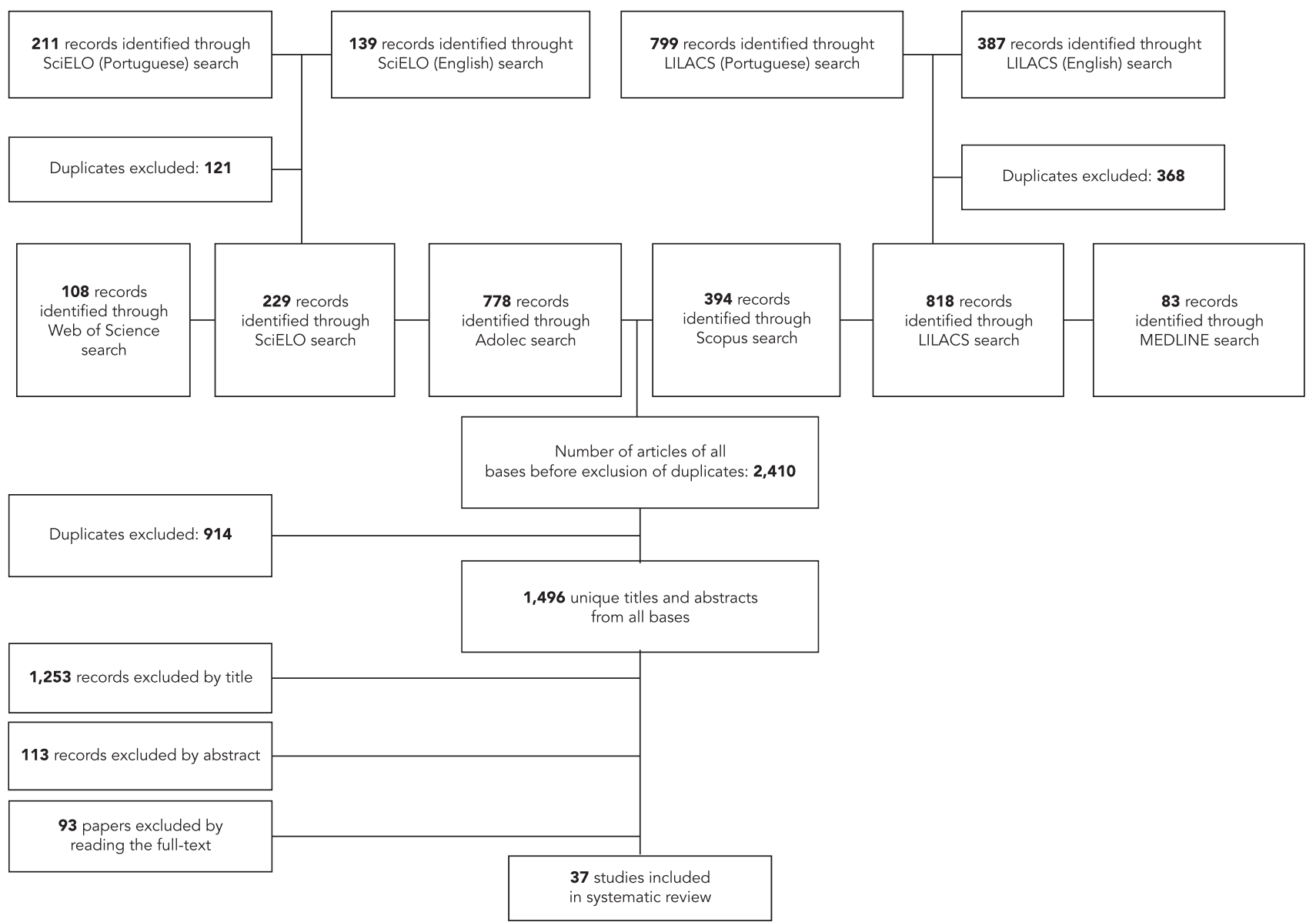

activity was a covariate in the study. Of the 37 studies, 35 were cross-sectional studies and two included phases of cohort studies, which had a cross-sectional analysis.

The characteristics of the studies are shown in Table 1. Most studies were conducted in the Southern region (19 studies), followed by the Southeastern and Northeastern regions (9 studies each). Only one study was performed in the Northern region. We did not find any studies that were carried out in the Central-Western region.

Most of the studies were based on schools with only six based on households and two on specific population groups (trainees from a university and adolescents from families observed at a Primary Care Unit as well as from hypertensive families).
The sample size ranged from 49 to 5,028 teenagers; 12 of the 37 studies were performed with samples sizes of up to 500 individuals, 11 with sample sizes between 500 and 1,000 adolescents and 14 with sample sizes of more than 1,000 individuals.

Inall studies, physical inactivity was measured indirectly (i.e. based on information provided by individuals). Questionnaires were the most commonly used instruments. However, some studies used daily logs to obtain information. Although most studies used previously validated questionnaires, 14 studies used questionnaires unique to their investigation.

There was a large amount of diversity in the areas considered in the assessment of physical activity. Some studies considered only vigorous 
Table 1

Characteristics of included studies

\begin{tabular}{|c|c|c|c|c|c|c|c|c|c|c|}
\hline Reference & $\begin{array}{l}\text { Data } \\
\text { collection } \\
\text { year }\end{array}$ & City (Region) & Base & Sampling & $\begin{array}{l}\text { Assessment } \\
\text { method }\end{array}$ & $\begin{array}{l}\text { Areas of } \\
\text { physical } \\
\text { activity } \\
\text { considered }\end{array}$ & $\begin{array}{l}\text { Cut-off point } \\
\text { for inactivity }\end{array}$ & $\%$ male & $\begin{array}{l}\text { Age } \\
\text { group } \\
\text { (years) }\end{array}$ & Total (n) \\
\hline Adami et al. 38 & 2004 & $\begin{array}{c}\text { Florianópolis } \\
\text { (South) }\end{array}$ & $\begin{array}{l}\text { School- } \\
\text { based }\end{array}$ & Non-random & $\begin{array}{l}\text { Questionnaire } \\
\text { (Pate et al. 39) }\end{array}$ & $\begin{array}{l}\text { Travel, } \\
\text { leisure and } \\
\text { sports }\end{array}$ & 12 points & 45.0 & $11-18$ & 242 \\
\hline Araújo et al. 40 & 2007 & $\begin{array}{c}\text { Fortaleza } \\
\text { (Northeast) }\end{array}$ & $\begin{array}{l}\text { School- } \\
\text { based }\end{array}$ & Non-random & $\begin{array}{c}\text { Self- } \\
\text { assessment } \\
\text { questionnaire }\end{array}$ & Not reported & $\begin{array}{c}\text { 3x/week, } \\
\text { minimum } 30 \\
\text { min/day }\end{array}$ & 42.7 & $12-17$ & 794 \\
\hline Arruda et al. 41 & - & Lages (South) & $\begin{array}{l}\text { School- } \\
\text { based }\end{array}$ & Cluster & $\begin{array}{c}\text { Records } \\
\text { (Bouchard et } \\
\text { al. }{ }^{24} \text { ) }\end{array}$ & N/A & $37 \mathrm{Kcal} / \mathrm{kg} /$ day & 100.0 & $10-17$ & 1,024 \\
\hline Bastos et al. 42 & 2005 & Pelotas (South) & Household & Cluster & $\begin{array}{c}\text { Self- } \\
\text { assessment } \\
\text { questionnaire }\end{array}$ & $\begin{array}{l}\text { Travel, } \\
\text { leisure } \\
\text { and sports } \\
\text { (minimum of } \\
10 \mathrm{~min} \text { ) }\end{array}$ & $300 \mathrm{~min} /$ week & 48.0 & 10-19 & 857 \\
\hline Bim et al. 43 & 2004 & $\begin{array}{l}\text { Maringá } \\
\text { (South) }\end{array}$ & $\begin{array}{l}\text { Specific } \\
\text { group }\end{array}$ & Non-random & $\begin{array}{l}\text { Questionnaire } \\
\text { (Pate RR, } \\
\text { translated and } \\
\text { modified by } \\
\text { Nahas } 44 \text { ) }\end{array}$ & $\begin{array}{l}\text { Travel, } \\
\text { leisure and } \\
\text { sports }\end{array}$ & $\begin{array}{c}\text { Inactive }+ \text { less } \\
\text { active }\end{array}$ & 68.5 & $16-17$ & 92 \\
\hline Castro et al. 45 & 2003 & $\begin{array}{l}\text { Rio de Janeiro } \\
\text { (Southeast) }\end{array}$ & $\begin{array}{l}\text { School- } \\
\text { based }\end{array}$ & Cluster & $\begin{array}{c}\text { Self- } \\
\text { assessment } \\
\text { questionnaire }\end{array}$ & $\begin{array}{l}\text { Travel, } \\
\text { leisure and } \\
\text { sports }\end{array}$ & $300 \mathrm{~min} /$ week & 47.2 & $13-18$ & 1,684 \\
\hline Ceschini et al. 46 & 2006 & $\begin{array}{l}\text { São Paulo } \\
\text { (Southeast) }\end{array}$ & $\begin{array}{l}\text { School- } \\
\text { based }\end{array}$ & Non-random & $\begin{array}{l}\text { Questionnaire } \\
\text { (Florindo et } \\
\text { al. 47) }\end{array}$ & $\begin{array}{l}\text { Travel, } \\
\text { leisure } \\
\text { and sports } \\
\text { (excludes } \\
\text { activity } \\
\text { during } \\
\text { school) }\end{array}$ & $300 \mathrm{~min} /$ week & 46.1 & $14-19$ & 775 \\
\hline Ceschini et al. 28 & 2006 & $\begin{array}{l}\text { São Paulo } \\
\text { (Southeast) }\end{array}$ & $\begin{array}{l}\text { School- } \\
\text { based }\end{array}$ & Cluster & $\begin{array}{l}\text { Questionnaire } \\
\text { (IPAQ - short } \\
\text { version) }\end{array}$ & $\begin{array}{l}\text { Intense and/ } \\
\text { or moderate } \\
\text { physical } \\
\text { activities } \\
\text { during or } \\
\text { outside of } \\
\text { school }\end{array}$ & $300 \mathrm{~min} /$ week & 47.4 & $14-19$ & 3,845 \\
\hline Chaves et al. 48 & $2006 / 2007$ & $\begin{array}{c}\text { Fortaleza } \\
\text { (Northeast) }\end{array}$ & $\begin{array}{l}\text { Specific } \\
\text { group }\end{array}$ & Non-random & $\begin{array}{c}\text { Self- } \\
\text { assessment } \\
\text { questionnaire }\end{array}$ & Not reported & $3 x /$ week & - & $12-18$ & 49 \\
\hline Dumith et al. 49 & 2008 & Pelotas (South) & Household & Census & $\begin{array}{c}\text { Self- } \\
\text { assessment } \\
\text { questionnaire }\end{array}$ & $\begin{array}{l}\text { Travel, } \\
\text { leisure and } \\
\text { sports }\end{array}$ & $300 \mathrm{~min} /$ week & 49.0 & $14-15$ & 4,325 \\
\hline
\end{tabular}

(continues) 
Table 1 (continued)

\begin{tabular}{|c|c|c|c|c|c|c|c|c|c|c|}
\hline Reference & $\begin{array}{c}\text { Data } \\
\text { collection } \\
\text { year }\end{array}$ & City (Region) & Base & Sampling & $\begin{array}{l}\text { Assessment } \\
\text { method }\end{array}$ & $\begin{array}{l}\text { Areas of } \\
\text { physical } \\
\text { activity } \\
\text { considered }\end{array}$ & $\begin{array}{l}\text { Cut-off point } \\
\text { for inactivity }\end{array}$ & $\%$ male & $\begin{array}{l}\text { Age } \\
\text { group } \\
\text { (years) }\end{array}$ & Total (n) \\
\hline Enes et al. 26 & 2005 & $\begin{array}{c}\text { Piedade } \\
\text { (Southeast) }\end{array}$ & $\begin{array}{l}\text { School- } \\
\text { based }\end{array}$ & Cluster & $\begin{array}{l}\text { Questionnaire } \\
\text { (Florindo et } \\
\text { al. }{ }^{47} \text { ) }\end{array}$ & $\begin{array}{c}\text { Travel, } \\
\text { leisure } \\
\text { and sports } \\
\text { (excludes } \\
\text { activity } \\
\text { during } \\
\text { school) }\end{array}$ & $300 \mathrm{~min} /$ week & 37.0 & $10-14$ & 105 \\
\hline Farias et al. 29 & 2002 & $\begin{array}{c}\text { Porto Velho } \\
\text { (North) }\end{array}$ & $\begin{array}{l}\text { School- } \\
\text { based }\end{array}$ & Non-random & $\begin{array}{l}\text { Questionnaire } \\
\text { (IPAQ) }\end{array}$ & $\begin{array}{l}\text { Intense and/ } \\
\text { or moderate } \\
\text { physical } \\
\text { activities } \\
\text { during or } \\
\text { outside of } \\
\text { school }\end{array}$ & $300 \mathrm{~min} /$ week & 50.8 & $11-14$ & 303 \\
\hline Farias Jr. 50 & 2001 & $\begin{array}{c}\text { Florianópolis } \\
\text { (South) }\end{array}$ & $\begin{array}{l}\text { School- } \\
\text { based }\end{array}$ & Cluster & $\begin{array}{c}\text { Records } \\
\text { (Bouchard et } \\
\text { al. }{ }^{24)}\end{array}$ & $N / A$ & $37 \mathrm{Kcal} / \mathrm{kg} /$ day & 49.41 & $12-18$ & 1,949 \\
\hline Farias Jr. et al. 51 & 2005 & $\begin{array}{c}\text { João Pessoa } \\
\text { (Northeast) }\end{array}$ & $\begin{array}{l}\text { School- } \\
\text { based }\end{array}$ & Cluster & Records & $N / A$ & $37 \mathrm{Kcal} / \mathrm{kg} /$ day & 44.1 & 14-18 & 2,768 \\
\hline Freitas et al. 52 & 2007 & $\begin{array}{c}\text { Fortaleza } \\
\text { (Northeast) }\end{array}$ & $\begin{array}{l}\text { School- } \\
\text { based }\end{array}$ & Non-random & $\begin{array}{c}\text { Self- } \\
\text { assessment } \\
\text { questionnaire }\end{array}$ & Not reported & $\begin{array}{c}\text { 3x/week, } \\
\text { minimum } 30 \\
\text { min/day }\end{array}$ & 44.6 & $12-17$ & 307 \\
\hline Guedes et al. 53 & 1998 & $\begin{array}{l}\text { Londrina } \\
\text { (South) }\end{array}$ & $\begin{array}{l}\text { School- } \\
\text { based }\end{array}$ & Non-random & $\begin{array}{c}\text { Records } \\
\text { (Bouchard et } \\
\text { al. }{ }^{24} \text { ) }\end{array}$ & $N / A$ & $37 \mathrm{Kcal} / \mathrm{kg} /$ day & 44.1 & 15-18 & 281 \\
\hline Guedes et al. 54 & 2003 & $\begin{array}{l}\text { Londrina } \\
\text { (South) }\end{array}$ & $\begin{array}{l}\text { School- } \\
\text { based }\end{array}$ & Non-random & $\begin{array}{c}\text { Records } \\
\text { (Bouchard et } \\
\text { al. } 24 \text { ) }\end{array}$ & $N / A$ & $37 \mathrm{Kcal} / \mathrm{kg} /$ day & 45.6 & 15-18 & 452 \\
\hline Guedes et al. 55 & $2004 / 2005$ & $\begin{array}{c}\text { Fortaleza } \\
\text { (Northeast) }\end{array}$ & $\begin{array}{l}\text { School- } \\
\text { based }\end{array}$ & Non-random & $\begin{array}{c}\text { Self- } \\
\text { assessment } \\
\text { questionnaire }\end{array}$ & Not reported & $\begin{array}{c}\text { 3x/week, } \\
\text { minimum } 30 \\
\text { min/day }\end{array}$ & 53.6 & $12-18$ & 56 \\
\hline Hallal et al. 5 & $2004 / 2005$ & Pelotas (South) & Household & Census & $\begin{array}{c}\text { Self- } \\
\text { assessment } \\
\text { questionnaire }\end{array}$ & $\begin{array}{l}\text { Travel and } \\
\text { leisure } \\
\text { (excludes } \\
\text { activity } \\
\text { during } \\
\text { school) }\end{array}$ & $300 \mathrm{~min} /$ week & 48.7 & $10-12$ & 4,451 \\
\hline Larcarotte et al. 56 & 1999-2001 & $\begin{array}{l}\text { São Paulo } \\
\text { (Southeast) }\end{array}$ & $\begin{array}{l}\text { School- } \\
\text { based }\end{array}$ & Cluster & $\begin{array}{c}\text { Self- } \\
\text { assessment } \\
\text { questionnaire }\end{array}$ & Not reported & $\begin{array}{c}\text { Does not } \\
\text { participate in } \\
\text { any physical } \\
\text { activity }\end{array}$ & 49.5 & $10-19$ & 2,125 \\
\hline $\begin{array}{l}\text { Magalhães } \\
\text { et al. } 57\end{array}$ & 1996/1997 & N/A & Household & Cluster & $\begin{array}{c}\text { Self- } \\
\text { assessment } \\
\text { questionnaire }\end{array}$ & $\begin{array}{l}\text { Leisure- } \\
\text { based } \\
\text { physical } \\
\text { activity }\end{array}$ & $\begin{array}{c}\text { Does not } \\
\text { participate } \\
\text { in any leisure } \\
\text { physical } \\
\text { activity }\end{array}$ & 52.39 & $15-19$ & $\begin{array}{c}1,027+ \\
824\end{array}$ \\
\hline
\end{tabular}

(continues) 
Table 1 (continued)

\begin{tabular}{|c|c|c|c|c|c|c|c|c|c|c|}
\hline Reference & $\begin{array}{c}\text { Data } \\
\text { collection } \\
\text { year }\end{array}$ & City (Region) & Base & Sampling & $\begin{array}{l}\text { Assessment } \\
\text { method }\end{array}$ & $\begin{array}{l}\text { Areas of } \\
\text { physical } \\
\text { activity } \\
\text { considered }\end{array}$ & $\begin{array}{l}\text { Cut-off point } \\
\text { for inactivity }\end{array}$ & $\%$ male & $\begin{array}{c}\text { Age } \\
\text { group } \\
\text { (years) }\end{array}$ & Total (n) \\
\hline Marini et al. 30 & - & $\begin{array}{l}\text { Londrina } \\
\text { (South) }\end{array}$ & $\begin{array}{l}\text { School- } \\
\text { based }\end{array}$ & Non-random & $\begin{array}{c}\text { Questionnaire } \\
\text { (IPAQ - short } \\
\text { version) }\end{array}$ & $\begin{array}{c}\text { Intense and/ } \\
\text { or moderate } \\
\text { physical } \\
\text { activities } \\
\text { during or } \\
\text { outside of } \\
\text { school }\end{array}$ & $\begin{array}{c}\text { Insufficiently } \\
\text { active + } \\
\text { sedentary }\end{array}$ & 45.7 & - & 92 \\
\hline Melo et al. 31 & - & $\begin{array}{l}\text { Belford Roxo } \\
\text { (Southeast) }\end{array}$ & $\begin{array}{l}\text { School- } \\
\text { based }\end{array}$ & Non-random & $\begin{array}{c}\text { Questionnaire } \\
\text { (IPAQ - short } \\
\text { version) }\end{array}$ & $\begin{array}{c}\text { Intense and/ } \\
\text { or moderate } \\
\text { physical } \\
\text { activities } \\
\text { during or } \\
\text { outside of } \\
\text { school }\end{array}$ & $\begin{array}{c}\text { Insufficiently } \\
\text { active }+ \\
\text { sedentary }\end{array}$ & 23.7 & $15-19$ & 93 \\
\hline Mendes et al. 58 & $2004 / 2005$ & $\begin{array}{c}\text { Recife } \\
\text { (Northeast) }\end{array}$ & $\begin{array}{l}\text { School- } \\
\text { based }\end{array}$ & Non-random & $\begin{array}{l}\text { Method not } \\
\text { mentioned }\end{array}$ & Not reported & $300 \mathrm{~min} /$ week & 41.0 & $14-19$ & 421 \\
\hline Moraes et al. 32 & - & $\begin{array}{l}\text { Maringá } \\
\text { (South) }\end{array}$ & $\begin{array}{l}\text { School- } \\
\text { based }\end{array}$ & Cluster & $\begin{array}{c}\text { Questionnaire } \\
\text { (IPAQ - short } \\
\text { version) }\end{array}$ & $\begin{array}{c}\text { Intense and/ } \\
\text { or moderate } \\
\text { physical } \\
\text { activities } \\
\text { during or } \\
\text { outside of } \\
\text { school }\end{array}$ & $300 \mathrm{~min} /$ week & 45.5 & $14-18$ & 991 \\
\hline $\begin{array}{l}\text { Oehlschlaeger } \\
\text { et al. } 59\end{array}$ & - & Pelotas (South) & Household & Cluster & $\begin{array}{c}\text { Self- } \\
\text { assessment } \\
\text { questionnaire }\end{array}$ & $\begin{array}{c}\text { Travel, } \\
\text { leisure and } \\
\text { sports }\end{array}$ & $\begin{array}{c}3 x / \text { week, } \\
\text { minimum } 20 \\
\text { min/day }\end{array}$ & 48.2 & $15-18$ & 960 \\
\hline Pelegrini et al. 33 & 2007 & $\begin{array}{c}\text { Florianópolis } \\
\text { (South) }\end{array}$ & $\begin{array}{l}\text { School- } \\
\text { based }\end{array}$ & Non-random & $\begin{array}{c}\text { Questionnaire } \\
\text { (IPAQ - short } \\
\text { version) }\end{array}$ & $\begin{array}{c}\text { Intense and/ } \\
\text { or moderate } \\
\text { physical } \\
\text { activities } \\
\text { during or } \\
\text { outside of } \\
\text { school }\end{array}$ & $300 \mathrm{~min} /$ week & 32.9 & $14-18$ & 595 \\
\hline Romanzini et al. 34 & 2005 & $\begin{array}{l}\text { Londrina } \\
\text { (South) }\end{array}$ & $\begin{array}{l}\text { School- } \\
\text { based }\end{array}$ & Cluster & $\begin{array}{c}\text { Questionnaire } \\
\text { (IPAQ - short } \\
\text { version) }\end{array}$ & $\begin{array}{c}\text { Intense and/ } \\
\text { or moderate } \\
\text { physical } \\
\text { activities } \\
\text { during or } \\
\text { outside of } \\
\text { school }\end{array}$ & $300 \mathrm{~min} /$ week & 38.4 & $15-18$ & 664 \\
\hline Romero et al. 27 & 2004 & $\begin{array}{l}\text { Piracicaba } \\
\text { (Southeast) }\end{array}$ & $\begin{array}{l}\text { School- } \\
\text { based }\end{array}$ & Cluster & $\begin{array}{l}\text { Questionnaire } \\
\text { (Florindo et } \\
\text { al. 47) }\end{array}$ & $\begin{array}{c}\text { Travel, } \\
\text { leisure } \\
\text { and sports } \\
\text { (excludes } \\
\text { activity } \\
\text { during } \\
\text { school) }\end{array}$ & $300 \mathrm{~min} /$ week & 45.7 & $10-15$ & 328 \\
\hline
\end{tabular}

(continues) 
Table 1 (continued)

\begin{tabular}{|c|c|c|c|c|c|c|c|c|c|c|}
\hline Reference & $\begin{array}{l}\text { Data } \\
\text { collection } \\
\text { year }\end{array}$ & City (Region) & Base & Sampling & $\begin{array}{l}\text { Assessment } \\
\text { method }\end{array}$ & $\begin{array}{l}\text { Areas of } \\
\text { physical } \\
\text { activity } \\
\text { considered }\end{array}$ & $\begin{array}{l}\text { Cut-off point } \\
\text { for inactivity }\end{array}$ & $\%$ male & $\begin{array}{l}\text { Age } \\
\text { group } \\
\text { (years) }\end{array}$ & Total (n) \\
\hline Silva et al. 60 & 1997/1998 & $\begin{array}{c}\text { Niterói } \\
\text { (Southeats) }\end{array}$ & $\begin{array}{l}\text { School- } \\
\text { based }\end{array}$ & Cluster & $\begin{array}{l}\text { Questionnaire } \\
\text { (PAQ - C) }\end{array}$ & $\begin{array}{c}\text { Travel, } \\
\text { leisure } \\
\text { and sports } \\
\text { (moderate } \\
\text { and intense) }\end{array}$ & Score 3 & 37.8 & $14-15$ & 325 \\
\hline Santos et al. 11 & 2006 & $\begin{array}{l}\text { Curitiba } \\
\text { (South) }\end{array}$ & $\begin{array}{l}\text { School- } \\
\text { based }\end{array}$ & Cluster & $\begin{array}{c}\text { Self- } \\
\text { assessment } \\
\text { questionnaire }\end{array}$ & $\begin{array}{l}\text { Moderate } \\
\text { to intense } \\
\text { physical } \\
\text { activity } \\
\text { (minimum of } \\
60 \text { minutes) }\end{array}$ & $300 \mathrm{~min} /$ week & 40.31 & $14-18$ & 1,615 \\
\hline Silva et al. 22 & - & $\begin{array}{c}\text { Aracajú } \\
\text { (Northeast) }\end{array}$ & $\begin{array}{l}\text { School- } \\
\text { based }\end{array}$ & Non-random & $\begin{array}{l}\text { Questionnaire } \\
\text { (PAQ - C) }\end{array}$ & $\begin{array}{l}\text { Travel, } \\
\text { leisure and } \\
\text { sports }\end{array}$ & Score 3 & 43.02 & $\geq 13$ & 774 \\
\hline Silva et al. 61 & 2002 & $\begin{array}{l}\text { Santa Catarina } \\
\text { State (South) }\end{array}$ & $\begin{array}{l}\text { School- } \\
\text { based }\end{array}$ & Cluster & $\begin{array}{c}\text { Self- } \\
\text { assessment } \\
\text { questionnaire }\end{array}$ & $\begin{array}{c}\text { Travel, } \\
\text { leisure } \\
\text { and sports } \\
\text { (moderate } \\
\text { and intense) }\end{array}$ & $300 \mathrm{~min} /$ week & 40.7 & $15-19$ & 5,028 \\
\hline Campos et al. 62 & 2008 & $\begin{array}{l}\text { Curitiba } \\
\text { (South) }\end{array}$ & $\begin{array}{l}\text { School- } \\
\text { based }\end{array}$ & Cluster & $\begin{array}{c}\text { Records } \\
\text { (Bouchard et } \\
\text { al. } 24 \text { ) }\end{array}$ & N/A & $37 \mathrm{Kcal} / \mathrm{kg} /$ day & 52.31 & $10-18$ & 497 \\
\hline Suñé et al. 63 & 2004 & $\begin{array}{c}\text { Capão da } \\
\text { Canoa (South) }\end{array}$ & $\begin{array}{l}\text { School- } \\
\text { based }\end{array}$ & Cluster & $\begin{array}{l}\text { Questionnaire } \\
\text { (Pate RR, } \\
\text { translated and } \\
\text { modified by } \\
\text { Nahas }{ }^{44)}\end{array}$ & Not reported & 5 points & 49.8 & $11-13$ & 719 \\
\hline Tenório et al. 10 & 2006 & $\begin{array}{l}\text { ParáibaState } \\
\text { (Northeast) }\end{array}$ & $\begin{array}{l}\text { School- } \\
\text { based }\end{array}$ & Cluster & $\begin{array}{l}\text { Questionnaire } \\
\text { (version } \\
\text { translated and } \\
\text { adapted from } \\
\text { GSHS) }\end{array}$ & $\begin{array}{c}\text { Travel, } \\
\text { leisure } \\
\text { and sports } \\
\text { (moderate } \\
\text { and intense) }\end{array}$ & $300 \mathrm{~min} /$ week & 40.2 & $14-19$ & 4,210 \\
\hline $\begin{array}{l}\text { Vasconcelos } \\
\text { et al. } 64\end{array}$ & - & $\begin{array}{l}\text { São Mateus do } \\
\text { Sul (South) }\end{array}$ & $\begin{array}{l}\text { School- } \\
\text { based }\end{array}$ & Non-random & $\begin{array}{c}\text { Records } \\
\text { (Bouchard et } \\
\text { al. 24) }\end{array}$ & N/A & $\begin{array}{c}\text { Quartile of } \\
\text { the general } \\
\text { population: } \\
\text { Male: } \\
35.71 \mathrm{Kcal} / \\
\text { kg/day and } \\
\text { Female: } \\
38.74 \mathrm{Kcal} / \mathrm{kg} / \\
\text { day }\end{array}$ & 45.0 & $12-16$ & 240 \\
\hline
\end{tabular}

GSHS: Global School-based Student Health Survey; IPAQ: International Physical Activity Questionnaire; PAQ: Physical Activity Questionnaire. 
and/or moderate-intensity physical activities (during or outside of school), whereas other studies considered travel, leisure and sports activities as physical activities, excluding activities during school. Finally, some studies considered all travel, leisure and sports activities, whether during or outside of school, as physical activity. Some articles did not specify the areas considered in the analysis.

With respect to the cut-off points for classifying physical inactivity, the most commonly used cut-off was $<300$ minutes per week of moderate to vigorous activity. However, the cut-off values varied widely between studies.

The overall prevalence of physical inactivity, as well as the prevalence stratified by sex, are shown in Table 2 . There was a wide variation in these estimates: the lowest was $5.42 \% 10$, and the highest was $91 \% 11$.

The heterogeneity of the results expressed by the $\mathrm{I}^{2}$ statistic was $99 \%$. Prevalence rates ranged from $2 \%$ to $80 \%$ for male and from $14 \%$ to $91 \%$ for female subgroups. Among females, $69.7 \%$ of the prevalences were above $50 \%$ whereas, among men, $29.4 \%$ were above $50 \%$.

When we evaluated the characteristics of the studies as a source of heterogeneity in the results, using meta-regression models, we observed that the region in which the study was conducted partially explained the inconsistency among prevalence rates for inactivity, both for males $(p=0.08)$ and females $(p=0.02)$. Figure 2 shows that the South has the lowest prevalence of inactivity, whereas the North and Northeast have the highest estimated prevalences.

The figures representing gender-specific data in the meta-analysis were constructed from 34 studies. One study was exclusively conducted in male adolescents, and, thus, female-specific data were gleaned from 33 studies. Of the 37 studies in the systematic review, three did not have any information on the prevalence of physical inactivity according to sex and are therefore not represented in Figure 2.

In the female group, we identified other possible sources of heterogeneity through there was a decline in the average prevalence found in studies using non-random sampling compared with studies with random sampling (by cluster, $\mathrm{p}=0.02$ ) and among studies with sample sizes over 1,000 adolescents $(\mathrm{p}=0.07)$.

None of the other variables, for both of the overall population and stratified by gender, were associated with inter-study variations in the prevalence of physical inactivity.
Table 2

Prevalence rates of physical inactivity among Brazilian adolescents - total and by gender.

\begin{tabular}{|c|c|c|c|c|}
\hline \multirow[t]{2}{*}{ Study } & \multirow[t]{2}{*}{ Total } & \multicolumn{3}{|c|}{ Prevalence (\%) } \\
\hline & & Total & Male & Female \\
\hline Adami et al. 38 & 242 & 36.7 & 29.4 & 43.0 \\
\hline Araújo et al. 40 & 794 & 63.9 & - & - \\
\hline Arruda et al. 41 & 1,024 & 29.4 & 29.4 & - \\
\hline Bastos et al. 42 & 857 & 69.8 & 56.5 & 82.1 \\
\hline Bim et al. 43 & 92 & 31.5 & 22.2 & 51.7 \\
\hline Castro et al. 45 & 1,684 & 59.9 & 47.8 & 73.9 \\
\hline Ceschini et al. 46 & 775 & 64.3 & 61.6 & 66.5 \\
\hline Ceschini et al. 28 & 3,845 & 62.5 & 49.7 & 71.6 \\
\hline Chaves et al. 48 & 49 & 71.6 & - & - \\
\hline Dumith et al. 49 & 4,325 & 51.8 & 37.4 & 65.5 \\
\hline Enes et al. 26 & 105 & 18.0 & - & - \\
\hline Farias et al. 29 & 303 & 58.4 & 53.2 & 63.7 \\
\hline Farias Jr. 50 & 1,949 & 62.6 & 51.4 & 73.5 \\
\hline Farias Jr. et al. 51 & 2,768 & 55.9 & 45.5 & 64.2 \\
\hline Freitas et al. 52 & 307 & 67.4 & 32.4 & 67.6 \\
\hline Guedes et al. 53 & 281 & 56.6 & 46.0 & 65.0 \\
\hline Guedes et al. 54 & 452 & 49.1 & 41.9 & 55.4 \\
\hline Guedes et al. 55 & 56 & 19.6 & 3.3 & 38.4 \\
\hline Hallal et al. 5 & 4,451 & 58.2 & 49.0 & 67.0 \\
\hline Larcarotte et al. 56 & 2,125 & 23.2 & 14.4 & 32.1 \\
\hline \multirow[t]{2}{*}{ Magalhães et al. 57} & 1,027 & 59.4 & 40.6 & 80.0 \\
\hline & 824 & 58.9 & 46.9 & 70.7 \\
\hline Marini et al. 30 & 92 & 8.6 & 2.4 & 14.0 \\
\hline Melo et al. 31 & 93 & 31.0 & 35.0 & 15.0 \\
\hline Mendes et al. 58 & 421 & 41.6 & - & - \\
\hline Moraes et al. 32 & 991 & 56.9 & 55.7 & 57.9 \\
\hline Oehlschlaeger et al. 59 & 960 & 39.0 & 22.2 & 54.5 \\
\hline Pelegrini et al. 33 & 595 & 25.4 & 21.9 & 27.1 \\
\hline Romanzini et al. 34 & 664 & 39.2 & 33.3 & 42.8 \\
\hline Romero et al. 27 & 328 & 54.9 & 35.0 & 65.0 \\
\hline Silva et al. 60 & 325 & 91.0 & 85.0 & 94.0 \\
\hline Santos et al. 11 & 1,615 & 85.3 & 77.9 & 90.9 \\
\hline Silva et al. 22 & 774 & 83.1 & 74.8 & 89.3 \\
\hline Silva et al. 61 & 5,028 & 28.5 & 27.9 & 28.8 \\
\hline Campos et al. 62 & 497 & 19.7 & 17.3 & 22.6 \\
\hline Suñé et al. 63 & 719 & 5.4 & - & - \\
\hline Tenório et al. 10 & 4,210 & 65.1 & 57.6 & 70.2 \\
\hline Vasconcelos et al. 64 & 240 & 25.0 & 25.0 & 25.0 \\
\hline
\end{tabular}


Figure 2

Forest plot of the prevalence rates of physical inactivity among Brazilian adolescents, by region and gender.

2a) Male prevalence

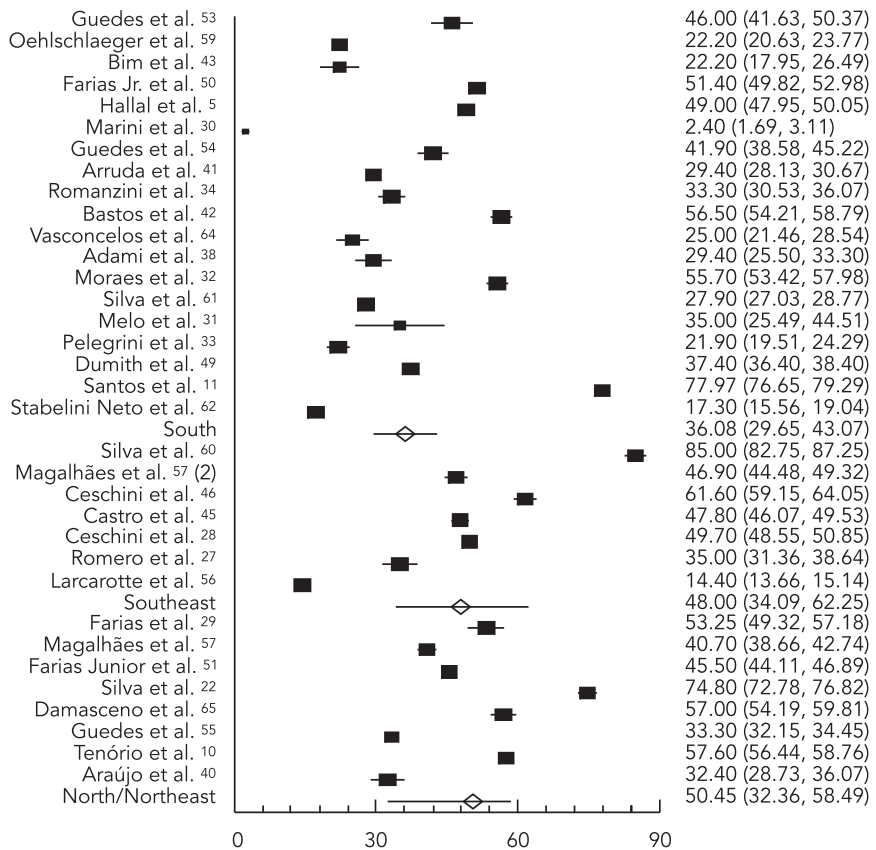

2b) Female prevalence

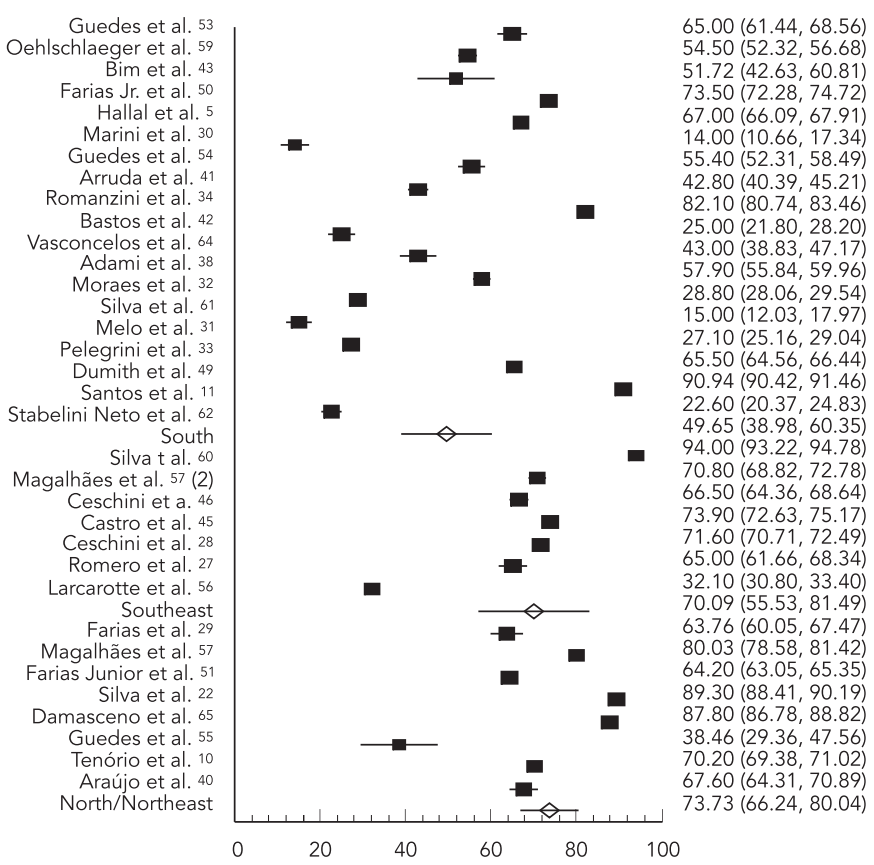




\section{Discussion}

This systematic review showed a wide range in prevalence of physical inactivity, as well as in the methods used to assess it, in studies conducted among Brazilian adolescents. We observed that the majority of studies were conducted in the Southern region. Although we investigated the profile of physical inactivity by region, this was not sufficient to explain the heterogeneity of the findings.

Most studies found a higher prevalence of physical inactivity among women, similar to findings in international studies 12,13,14. Researchers have reported that this gender difference is related to cultural, behavioral, psychological and maturation factors 13,15 . However, there have been no studies conducted in Brazil to assess the possible explanations for this difference.

Despite the progress in developing tools to assess physical activity, there are still many limitations in these methods, which are amplified among children and adolescents. The patterns of physical activity among young people can be influenced by cognitive, physiological and biomechanical changes that occur during development 16,17. Physical activity among young people tends to be intermittent and of a more variable intensity than among adults, usually consisting of activities that involve less planning and less time 18,19 .

A major challenge for researchers is to obtain a reliable and valid instrument to measure physical activity 20 . For logistical reasons, questionnaires are used in most studies although efforts are being made to use direct measurements more frequently. Therefore, accurate and valid methods for measuring physical activity among adolescents, as well as among other age groups, are needed to determine the dose-response associations between physical activity and health, in order to monitor temporal trends in populations and to make cross-cultural comparisons also determining the effect of interventions 21 .

In Brazil, there are no standardised instruments, direct or indirect, to estimate physical inactivity among young people 22 . All studies have used indirect methods to assess physical activity (i.e., methods that use reports from the individuals themselves). The accuracy of self-reporting is influenced by the respondent's ability to accurately recall all relevant activities, retrospectively. Therefore, indirect methods are subject to recall bias 23 .

Only seven studies used physical activity logs, an instrument in which the individual registers any and all activities performed during the day in short time intervals (typically 15 -minute peri- ods) 24. Most researchers opted to use questionnaires, of which 16 were validated and 14 were unique to the individual study. Although validation studies have low correlation values between the questionnaires evaluated and a gold standard, their use is still recommended over unique, non-validated questionnaires 25 . The use of validated instruments allows for comparisons between different studies.

The use of different methods to assess physical activity could affect the results of the studies. Some authors found higher prevalences when their questionnaires included all domains (commuting to work, leisure-time and sports) and different environments of physical activity (inside or outside school) when compared with other studies that excluded some domains or activities at school. For example, some authors defined physical activity as the practice of sports outside school as well as going to school by bike or walking 26,27. Other authors considered any kind of vigorous and/or moderate physical activity, inside or outside school, in a structured way or not $28,29,30,31,32,33,34$. The lack of a homogeneous definition of physical activity is an important limitation for pooling, or even comparing, results among different studies.

Another factor that complicates comparisons between the studies of Brazilian adolescents is the lack of standardization for the cut-off point used to classify individuals as "inactive". Most public health authorities agree that adolescents should accumulate at least 60 minutes of moderate-to-vigorous physical activity at least five days a week $7,35,36$, resulting in a cut-off point of 300 minutes per week. However, when addressing cut-off points, studies had noted variability using different values for this classification.

The difficulties in explaining the observed heterogeneity can be attributed to the dispersed nature and small number of studies related to the characteristics analysed. For example, although 16 studies used the same cut-off point for inactivity, $300 \mathrm{~min} /$ week, these 16 studies used different questionnaires and considered different areas of physical activity.

Sisson \& Katzmarzyk ${ }^{37}$ performed a review of studies presenting national prevalences of physical activity (more than $300 \mathrm{~min} /$ week). In their review they reported that women were more inactive than men, considering both adults $(>18$ years) and adolescents ( $<18$ years). In studies with young subjects, the prevalence of physical inactivity varied widely among populations. The countries with highest prevalences were Belgium, France, and Tonga.

To the best of our knowledge, there had been no previously published studies using systematic 
review and meta-analysis tools to investigate the prevalence of physical activity/inactivity among adolescents.

\section{Conclusion}

Despite the noted challenges, we believe that this study is the first effort to systematise information regarding physical inactivity, which is largely relevant to the health of adolescents in Brazil. This review highlights the urgent need to use standardised and validated methods in order to measure physical activity. As such, we shall be able to identify specific groups for intervention action, increasing their physical activity and its beneficial effects.

\section{Resumo}

Foram realizadas revisão sistemática e meta-análise para investigar a prevalência de inatividade física entre adolescentes no Brasil. Identificou-se artigos publicados até agosto de 2010. A busca foi realizada em 6 bases de dados eletrônicas, não tendo sido utilizados quaisquer limites. Gráficos do tipo forest-plots foram gerados usando-se a prevalência de inatividade física estratificada por região e sexo. Modelos de metarregressão foram ajustados para identificar as possiveis fontes de heterogeneidade nas estimativas. Dos 1.496 artigos identificados inicialmente, 37 foram considerados adequados. As taxas de prevalência variaram de $2 \%$ a $80 \%$ para o gênero masculino e de $14 \%$ para 91\% para o feminino. As menores taxas de prevalência foram encontradas na Região Sul, e as maiores nas regiões Norte e Nordeste. Os métodos utilizados para avaliar a inatividade física em adolescentes brasileiros também diferiram entre os estudos. Essa variação demonstra a necessidade de métodos padronizados e validados para medir a atividade física na pesquisa epidemiológica.

Atividade Motora; Adolescente; Metanálise

\section{Contributors}

L. A. Barufaldi, G. A. Abreu participated in the literature search, article selection, extraction and analysis of the data and in the drafting of the manuscript. E. S. F. Coutinho participated in the analysis of the data and in the drafting of the manuscript. K. V. Bloch participated in article selection, analysis of the data and in the drafting of the manuscript.

\section{Acknowledgments}

We would like to thank FAPERJ (process no. E26/102.797/2010) for providing a doctoral fellowship to L. A. Barufaldi and CNPq (process no. 381768/2010-4) for providing a research grant to G. A. Abreu. E. S. F. Coutinho was partially supported by CNPq (process no. 302269/2008-8). K. V. Block was partially supported by CNPq (process no. 303594/2009-8). 


\section{References}

1. Langness A, Richter M, Hurrelmann K. Health behaviour in school-aged children: results of the international study "Health Behavior in School-aged Children”. Gesundheitswesen 2005; 4:196-204.

2. Dowdell EB, Santucci ME. Health risk behavior assessment: nutrition, weight, and tobacco use in one urban seventh-grade class. Public Health Nurs 2004; 21:128-36.

3. Instituto Brasileiro de Geografia e Estatística. Pesquisa Nacional de Saúde do Escolar, 2009. Rio de Janeiro: Instituto Brasileiro de Geografia e Estatística; 2009.

4. Ministério da Saúde. Escolas promotoras de saúde: experiências do Brasil. Brasília: Ministério da Saúde; 2006. (Série Promoção da Saúde, 6).

5. Hallal PC, Bertoldi AD, Gonçalves H, Victora CG. Prevalência de sedentarismo e atores associados em adolescentes de 10-12 anos de idade. Cad Saúde Pública 2006; 22:1277-87.

6. Guthold R, Ono T, Strong KL, Chatterji S, Morabia A. Worldwide variability in physical inactivity a 51country survey. Am J Prev Med 2008; 34:486-94.

7. United States Department of Health and Human Services. Physical Activity Guidelines Advisory Committee Report, 2008. Washington DC: United States Department of Health and Human Services; 2008.

8. Dumith SC. Physical activity in Brazil: a systematic review. Cad Saúde Pública 2009; 25 Suppl 3: S415-26.

9. Higgins JP, Thompson SG, Deeks JJ, Altman DG. Measuring inconsistency in meta-analyses. BMJ 2003; 327:557-60

10. Tenório MCM, Barros MVG, Tassitano RM, Bezerra J, Tenório JM, Hallal PC. Atividade física e comportamento sedentário em adolescentes estudantes do ensino médio. Rev Bras Epidemiol 2010; 13: 105-17.

11. Santos MS, Hino AAF, Reis RS, Rodriguez-Añez CR. Prevalência de barreiras para a prática de atividade física em adolescentes. Rev Bras Epidemiol 2010; 13:94-104.

12. Crocker PR, Bailey DA, Faulkner RA, Kowalski KC, McGrath R. Measuring general levels of physical activity: preliminary evidence for the Physical Activity Questionnaire for Older Children. Med Sci Sports Exerc 1997; 29:1344-9.

13. Thompson AM, Baxter-Jones AD, Mirwald RL, Bailey DA. Comparison of physical activity in male and female children: does maturation matter? Med Sci Sports Exerc 2003; 35:1684-90.

14. Nader PR, Bradley RH, Houts RM, McRitchie SL, O'Brien M. Moderate-to-vigorous physical activity from ages 9 to 15 years. JAMA 2008; 300:295-305.

15. Nitzan Kaluski D, Demem Mazengia G, Shimony T, Goldsmith R, Berry EM. Prevalence and determinants of physical activity and lifestyle in relation to obesity among schoolchildren in Israel. Public Health Nutr 2009; 12:774-82.

16. Sallis JF. Self-report measures of children's physical activity. J Sch Health 1991; 61:215-9.
17. Armstrong N, Welsman JR. Peak oxygen uptake in relation to growth and maturation in 11- to 17-year-old humans. Eur J Appl Physiol 2001; 85: 546-51.

18. Berman N, Bailey R, Barstow T, Cooper DM. Spectral and bout detection analysis of physical activity patterns in healthy, prepubertal boys and girls. Am J Hum Biol 1998; 10:289-97.

19. Baquet G, Stratton G, Van Praagh E, Berthoin S. Improving physical activity assessment in prepubertal children with high-frequency accelerometry monitoring: a methodological issue. Prev Med 2007; 44:143-7.

20. Farias Jr. JC, Lopes AS, Florindo AA, Hallal PC. Validade e reprodutibilidade dos instrumentos de medida da atividade física do tipo self-report em adolescentes: uma revisão sistemática. Cad Saúde Pública 2010; 26:1669-91.

21. Wareham NJ, Rennie KL. The assessment of physical activity in individuals and populations: why try to be more precise about how physical activity is assessed? Int J Obes Relat Metab Disord 1998; 22 Suppl 2:S30-8.

22. Silva DAS, Lima JO, Silva RJS, Prado RL. Nível de atividade física e comportamento sedentário em escolares. Rev Bras Cineantropom Desempenho Hum 2009; 11:299-306.

23. Pate RR. Physical activity assessment in children and adolescents. Crit Rev Food Sci Nutr 1993; 33:321-6.

24. Bouchard CA, Tremblay C, Leblanc G, Lortie R, Savard R, Theriault G. A method to assess energy expenditure in children and adults. Am J Clin Nutr 1983; 37:461-7.

25. Ekelund U, Tomkinson GR, Armstrong N. What proportion of youth are physically active? Measurement issues, levels and recent time trends. Br J Sports Med 2011; 45:859-65.

26. Enes CC, Pegolo GE, Silva MV. Influência do consumo alimentar e do padrão de atividade física sobre o estado nutricional de adolescentes de Piedade, São Paulo. Rev Paul Pediatr 2009; 27:265-71.

27. Romero A, Slater B, Florindo AA, Latorre MRDO, Cezar C, Silva MV. Determinantes do índice de massa corporal em adolescentes de escolas públicas de Piracicaba, São Paulo. Ciênc Saúde Coletiva 2010; 15:141-9.

28. Ceschini FL, Andrade DR, Oliveira LC, Araújo Júnior JF, Matsudo VKR. Prevalência de inatividade física e fatores associados em estudantes do ensino médio de escolas públicas estaduais. J Pediatr (Rio J.) 2009; 85:301-6.

29. Farias ES, Salvador MRD. Antropometria, composição corporal e atividade física de escolares. Rev Bras Cineantropom Desempenho Hum 2005; 7:21-9.

30. Marini F, Oliveira AR, Guedes DP. Indicadores comportamentais associados à prática de atividade física e saúde em escolares do ensino médio. Rev Bras Ciênc Mov 2006; 14:63-70. 
31. Melo FAP, Oliveira FMF, Almeida MB. Nível de atividade física não identifica o nível de flexibilidade de adolescentes. Rev Bras Ativ Fís Saúde 2009; 14:48-54.

32. Moraes ACF, Fernandes CAM, Elias RGM, Nakashima ATA, Reichert FF, Falcão MC. Prevalência de inatividade física e fatores associados em adolescentes. Rev Assoc Med Bras 2009; 55:523-8.

33. Pelegrini A, Petroski EL. Inatividade física e sua associação com estado nutricional, insatisfação com a imagem corporal e comportamentos sedentários em adolescentes de escolas públicas. Rev Paul Pediatr 2009; 27:366-73.

34. Romanzini M, Reichert FF, Lopes AS, Petroski EL, Farias Jr. JC. Prevalência de fatores de risco cardiovascular em adolescentes. Cad Saúde Pública 2008; 24:2573-81.

35. Pate RR, Freedson PS, Sallis JF, Taylor WC, Sirard J, Trost SG, et al. Compliance with physical activity guidelines: prevalence in a population of children and youth. Ann Epidemiol 2002; 12:303-8.

36. Strong WB, Malina RM, Blimkie CJ, Daniels SR, Dishman RK, Gutin B, et al. Evidence based physical activity for school-age youth. J Pediatr 2005; 146:732-7.

37. Sisson SB, Katzmarzyk PT. International prevalence of physical activity in youth and adults. Obes Rev 2008; 9: 606-14.

38. Adami F, Frainer DES, Santos JS, Fernandes TC, Oliveira FR. Insatisfação corporal e atividade física em adolescentes da região continental de Florianópolis. Psicol Teor Pesq 2008; 24:143-9.

39. Pate RR, Pratt M, Blair SN, Haskel WL, Macera CA, Bouchard C, et al. Physical activity and public health: a recommendation from the Centers for Disease Control and Prevention and the American College of Sports Medicine. JAMA 1995; 273:402-7.

40. Araújo MFM, Almeida LS, Silva PCV, Vasconcelos HCA, Lopes MVO, Damasceno MMC. Sobrepeso entre adolescentes de escolas particulares de Fortaleza, CE, Brasil. Rev Bras Enferm 2010; 63:623-8.

41. Arruda ELM, Lopes AS. Gordura corporal, nível de atividade física e hábitos alimentares de adolescentes da região serrana de Santa Catarina, Brasil. Rev Bras Cineantropom Desempenho Hum 2007; 9:5-11.

42. Bastos JP, Araújo CLP, Hallal PC. Prevalence of insufficient physical activity and associated factors. J Phys Act Health 2008; 5:777-94.

43. Bim RH, Junior NN. Aptidão física relacionada à saúde de adolescentes estagiários da Universidade Estadual de Maringá. Acta Sci Health Sci 2005; 27:77-85.

44. Nahas MV. Atividade física, saúde e qualidade de vida: conceitos e sugestões para um estilo de vida ativo. 3a Ed. Londrina: Midiograf; 2003.

45. Castro IRR, Cardoso LO, Engstrom EM, Levy RB, Monteiro CA. Vigilância de fatores de risco para doenças não transmissíveis entre adolescentes: a experiência da cidade do Rio de Janeiro, Brasil. Cad Saúde Pública 2008; 24:2279-88.

46. Ceschini FL, Florindo AA, Benicio MHD. Nível de atividade física em adolescentes de uma região de elevado índice de vulnerabilidade juvenil. Rev Bras Ciênc Mov 2007; 15:67-78.
47. Florindo AA, Romero A, Peres SV, Silva MV, Slater B. Development and validation of a physical activity assessment questionnaire for adolescents. Rev Saúde Pública 2006; 40:802-9.

48. Chaves ES, Araújo TL, Chaves DBR, Costa AGS, Oliveira ARS, Alves FEC. Crianças e adolescentes com história familiar de hipertensão arterial: indicadores de risco cardiovasculares. Acta Paul Enferm 2009; 22:793-9.

49. Dumith SC, Domingues MR, Gigante DP, Hallal PC, Menezes AMB, Kohl HW. Prevalence and correlates of physical activity among adolescents from Southern Brazil. Rev Saúde Pública 2010; 44: 457-67.

50. Farias Jr. JC. Prevalência e fatores de influência para inatividade física em adolescentes. Rev Bras Ciênc Mov 2006; 14:63-70.

51. Farias Jr. JC, Mendes JKF, Barbosa DBM. Associação entre comportamentos de risco à saúde em adolescentes. Rev Bras Cineantropom Desempenho Hum 2007; 9:250-6.

52. Freitas RWJF, Silva ARV, Araújo MFM, Marinho NBP, Damasceno MMC, Oliveira MR. Prática de atividade física por adolescentes de Fortaleza, CE, Brasil. Rev Bras Enferm 2010; 63:410-5.

53. Guedes DP, Guedes JERP, Barbosa DS, Oliveira JA. Níveis de prática de atividade física habitual em adolescentes. Rev Bras Med Esporte 2001; 7: 187-99.

54. Guedes DP, Guedes JERP, Barbosa DS, Oliveira JA, Stanganelli LCR. Fatores de Risco cardiovasculares em adolescentes: indicadores biológicos e comportamentais. Arq Bras Cardiol 2006; 86:439-50.

55. Guedes NG, Moreira RP, Cavalcante TF, Araújo TL, Ximenes LB. Atividade física de escolares: análise segundo o modelo teórico de promoção de saúde de Pender. Rev Esc Enferm USP 2009; 43:774-80.

56. Lancarotte I, Nobre MR, Zanetta R, Polydoro M. Lifestyle and cardiovascular health in school adolescents from São Paulo. Arq Bras Cardiol 2010; 95:61-9.

57. Magalhães VC, Mendonça GAS. Prevalência e fatores associados a sobrepeso e obesidade em adolescentes de 15 a 19 anos das regiões Nordeste e Sudeste do Brasil, 1996 a 1997. Cad Saúde Pública 2003; 19 Suppl 1:S129-39.

58. Mendes MJFL, Alves JGB, Alves AV, Siqueira PP, Freire EFC. Associação de fatores de risco para doenças cardiovasculares em adolescentes e seus pais. Rev Bras Saúde Matern Infant 2006; 6 Suppl 1:S49-54.

59. Oehlschlaeger MHK, Pinheiro RT, Horta B, Gelatti C, San'Tana P. Prevalência e fatores associados ao sedentarismo em adolescentes de área urbana. Rev Saúde Pública 2004; 38:157-63.

60. Silva RCR, Malina RM. Nível de atividade física em adolescentes do Município de Niterói, Rio de Janeiro, Brasil. Cad Saúde Pública 2000; 16:1091-7.

61. Silva KS, Nahas MV, Peres KG, Lopes AS. Fatores associados à atividade física, comportamento sedentário e participação na Educação Física em estudantes do Ensino Médio em Santa Catarina, Brasil. Cad Saúde Pública 2009; 25:2187-200. 
62. Campos W, Stabelini Neto A, Bozza R, Ulbrich AZ, Bertin RL, Mascarenhas LPG, et al. Atividade física, consumo de lipídios e fatores de risco para aterosclerose em adolescentes. Arq Bras Cardiol 2010; 94:601-7.

63. Suñé FR, Dias-da-Costa JS, Olinto MTA, Pattussi MP. Prevalência e fatores associados para sobrepeso e obesidade em escolares de uma cidade no Sul do Brasil. Cad Saúde Pública 2007; 23:1361-71.

64. Vasconcelos IQA, Stabelini A, Mascarenhas LPG, Bozza R, Ulbrich AZ, Campos W, et al. Fatores de risco cardiovascular em adolescentes com diferentes níveis de gasto energético. Arq Bras Cardiol 2008; 91:227-33.
65. Damasceno MMC, Freitas RWJF, Silva ARV, Almeida PC, Hissa MN. La práctica de actividad física entre los adolescentes de las escuelas públicas del estado en Fortaleza (Brasil). Apunts: Educación Física y Deportes 2009; 96:22-6.

Submitted on 10/Dec/2011

Final version resubmitted on 15/Feb/2012

Approved on 28/Fev/2012 